Pacific Journal of Mathematics

OSCILLATION CRITERIA FOR SECOND ORDER SELF 


\title{
OSCILLATION CRITERIA FOR SECOND ORDER SELF ADJOINT DIFFERENTIAL SYSTEMS
}

\author{
Garret J. Etgen and James F. Pawlowski
}

Let $\mathscr{H}$ be a Hilbert space and let $\mathscr{B}=\mathscr{B}(\mathscr{H}, \mathscr{H})$ be the Banach algebra of bounded linear operators from $\mathscr{H}$ to $\mathscr{H}$ with the usual operator norm. Let $\mathscr{S}$ be the subspace of $\mathscr{B}$ consisting of the self adjoint operators, and consider the second order differential system

$$
Y^{\prime \prime}+P(x) Y=0
$$

on $R^{+}=[0, \infty)$, where $P: R^{+} \rightarrow \mathscr{S}$ is continuous. Let $\mathscr{G}$ be the set of positive linear functionals on $\mathscr{B}$. The elements of $\mathscr{G}$ are used to derive oscillation criteria for this differential system. These criteria include a large number of well-known oscillation criteria for corresponding matrix differential systems and scalar equations. Extensions of the results to nonlinear differential systems and differential inequalities are also discussed.

Appropriate discussions of the concepts of differentiation and integration of functions from $R^{+}$to $\mathscr{B}$, as well as the existence and uniqueness of solutions $Y: R^{+} \rightarrow \mathscr{B}$ of (1), can be found in E. Hille's text [8, Chapters 4, 6, 9].

Let $Y=Y(x)$ be any solution of (1). It is easy to verify by differentiation that

$$
Y^{*} Y^{\prime}-Y^{* \prime} Y^{*}=C \quad \text { (constant) }
$$

on $R^{+}$(* denotes adjoint). The term conjoined (or prepared) is used to describe a solution $Y$ for which the constant operator $C$ in (2) is 0 . The solution $Y$ is nonsingular at $c, c \in R^{+}$, if

(i) The range of $Y(c)$ is $\mathscr{H}$, and

(ii) $Y(c)$ has a bounded inverse.

If one (or both) of these conditions fails to hold at $c$, then $Y$ is said to be singular at $c$. The solution $Y$ is nontrivial if there is at least one $c \in R^{+}$ at which $Y$ is nonsingular. In the case where $\mathscr{H}$ is finite dimensional it can be shown that a solution $Y$ of (1) is nontrivial and conjoined if and only if there exists a point $b \in R^{+}$such that

(i) $Y^{*}(b) Y^{\prime}(b)=Y^{* \prime}(b) Y(b)$, and

(ii) $Y^{*}(b) Y(b)+Y^{* \prime}(b) Y^{\prime}(b)$ is positive definite. 
T. L. Hayden and H. C. Howard [7, Theorem 1] have shown that the set of singular points of a solution $Y$ is closed. A nontrivial conjoined solution $Y$ of (1) is oscillatory if for each $a \in R^{+}$there is a point $c>a$ such that $Y(c)$ is singular. A conjoined solution $Y$ is nonoscillatory if there exists a subinterval $[b, \infty)$ of $R^{+}$on which $Y$ is nonsingular. The differential equation (1) is oscillatory if it has at least one nontrivial conjoined oscillatory solution; otherwise (1) is said to be nonoscillatory.

A linear functional $g$ on $\mathscr{B}$ is positive if $g\left(A^{*} A\right) \geqq 0$ for all $A \in \mathscr{B}$. A complete discussion of positive linear functionals on a Banach algebra is contained in the text [15] by C. E. Rickart. In particular, it is shown that if $g$ is a positive linear functional on $\mathscr{B}$, then

(i) $g$ is bounded (i.e., continuous),

(ii) $\|g\|=g(I)$ ( $I$ is the identity operator), and

(iii) $\left|g\left(A^{*} B\right)\right|^{2} \leqq g\left(A^{*} A\right) g\left(B^{*} B\right)$ for all $A, B \in \mathscr{B}$.

By letting $A=I$ in (iii), we have

$$
|g(B)|^{2} \leqq g(I) g\left(B^{*} B\right)
$$

for all $B \in \mathscr{B}$. Thus it follows that $g \neq 0$ if and only if $g(I)>0$. From this brief discussion it is easy to see that a nonzero linear functional $g$ on $\mathscr{B}$ is positive if and only if there exists a positive number $\rho=\rho(g)=$ $1 / g(I)$ such that

$$
g\left(B^{*} B\right) \geqq \rho|g(B)|^{2}
$$

for all $B \in \mathscr{B}$. Also, it should be clear that $g$ is a positive linear functional on $\mathscr{B}$ if and only if $g(A) \geqq 0$ for all $A \in \mathscr{B}$ such that $A$ is nonnegative definite. Finally, since a positive linear functional is continuous, we have

$$
g\left[\int_{a}^{x} A(t) d t\right]=\int_{a}^{x} g[A(t)] d t, \quad a, x \in R^{+},
$$

whenever $A: R^{+} \rightarrow \mathscr{B}$ is integrable, and

$$
g\left[B^{\prime}(x)\right]=\{g[B(x)]\}^{\prime}, \quad x \in R^{+},
$$

whenever $B: R^{+} \rightarrow \mathscr{B}$ is differentiable.

Let $\mathscr{G}$ be the set of positive linear functionals on $\mathscr{B}$. Specific examples of elements of $\mathscr{G}$ are obtained by associating with each vector $\alpha \in \mathscr{H}$ the linear functional $g_{\alpha}$ on $\mathscr{B}$ defined by

$$
g_{\alpha}(A)=(A \alpha, \alpha)
$$


for all $A \in \mathscr{B}$, where $(\cdot, \cdot)$ is the inner product on $\mathscr{H}$. It is easy to verify that $g_{\alpha}$ is positive. Also, if $g_{1}, g_{2}, \cdots, g_{k}$ are elements of $\mathscr{G}$ and $r_{1}, r_{2}, \cdots, r_{k}$ are nonnegative numbers, then $g=\sum_{i=1}^{k} r_{i} g_{\imath} \in \mathscr{G}$. In particular, $\mathscr{G}$ is a convex subset of the set of all bounded linear functionals on $\mathscr{B}$.

In this paper we shall use positive linear functionals on $\mathscr{B}$ to develop oscillation criteria for (1), and we shall show how these criteria include a large number of well-known oscillation criteria for matrix differential systems of the form (1), as well as for the scalar equation

$$
y^{\prime \prime}+p(x) y=0
$$

Extensions of our results to nonlinear differential systems and differential inequalities of the form (1) are discussed in the final section of the paper.

2. Oscillation criteria. Our first theorem gives an oscillation criterion for the differential equation (1). The method of proof and the manner in which positive functionals are used provide a model for the other results in the paper.

THEOREM 1. If there exists an element $g \in \mathscr{G}$ such that

$$
\lim _{x \rightarrow \infty} g\left[\int_{0}^{x} P(t) d t\right]=\infty
$$

then equation (1) is oscillatory.

Proof. This proof is based on the proof of a corresponding result for the scalar version (6) of equation (1) given by W. J. Coles [2].

Let $g$ be an element of $\mathscr{G}$ such that (7) holds, and suppose that (1) is nonoscillatory. Let $Y$ be nontrivial conjoined solution of (1). Then there exists a point $b \in R^{+}$such that $Y$ is nonsingular on $[b, \infty)$. Define the operator $Z$ by

$$
Z(x)=-Y^{\prime}(x) Y^{-1}(x) \quad \text { on } \quad[b, \infty) .
$$

The conjoined property of $Y$ implies that $Z$ is self adjoint. By differentiating $Z$ and using the fact that $Y$ is a solution of (1), we obtain

$$
Z^{\prime}(x)=P(x)+Z^{2}(x) \quad \text { on } \quad[b, \infty) .
$$

Therefore

$$
Z(x)=Z(b)+\int_{b}^{x} P(t) d t+\int_{b}^{x} Z^{2}(t) d t
$$


and

$$
g[Z(x)]=g[Z(b)]+g\left[\int_{b}^{x} P(t) d t\right]+g\left[\int_{b}^{x} Z^{2}(t) d t\right]
$$

Since $Z^{2}$ is nonnegative definite, $\int_{b}^{x} Z^{2}(t) d t$ is nonnegative definite and $g\left[\int_{b}^{x} Z^{2}(t) d t\right] \geqq 0$ on $[b, \infty)$. Thus

$$
g[Z(x)] \geqq g[Z(b)]+g\left[\int_{b}^{x} P(t) d t\right] \quad \text { on } \quad[b, \infty)
$$

It follows from the hypothesis (7) that $g\left[\int_{b}^{x} P(t)\right] d t \rightarrow \infty$ as $x \rightarrow \infty$, so that there exists $c \geqq b$ such that

$$
g[Z(b)]+g\left[\int_{b}^{x} P(t) d t\right]>0 \quad \text { on } \quad[c, \infty) .
$$

Therefore, from (8), $g[Z(x)]>g\left[\int_{b}^{x} Z^{2}(t) d t\right]$ and $g[Z(x)]>0$ on $[c, \infty)$.

Define the operator $W$ on $[b, \infty)$ by

$$
W(x)=\int_{b}^{x} Z^{2}(t) d t
$$

Then, by our results immediately above, together with the basic properties of the positive functional $g$, we have

$$
g[Z(x)]>g[W(x)]=\int_{b}^{x} g\left[Z^{2}(t)\right] d t \geqq \rho \int_{b}^{x}\{g[Z(t)]\}^{2} d t>0
$$

on $[c, \infty)$, where $\rho=1 / g(I)$ is the positive number associated with $g$ according to the characterization (4) of positive linear functionals.

Now $W^{\prime}(x)=Z^{2}(x)$. Therefore

$$
\{g[W(x)]\}^{\prime}=g\left[W^{\prime}(x)\right]=g\left[Z^{2}(x)\right] \geqq \rho\{g[Z(x)]\}^{2}>\rho\{g[W(x)]\}^{2},
$$

so that

$$
\frac{\{g[W(x)]\}^{\prime}}{\{g[W(x)]\}^{2}}>\rho \quad \text { on } \quad[c, \infty)
$$


It follows from this inequality that

$$
\frac{1}{g[W(c)]}-\frac{1}{g[W(x)]}>\rho(x-c)=\rho x-\rho c
$$

and

$$
\rho x-\rho c<\frac{1}{g[W(c)]} \quad \text { on } \quad[c, \infty)
$$

since $g[W(x)]>0$. However, the expression $\rho x-\rho c$ is obviously not bounded on $[c, \infty)$, and hence we have a contradiction which establishes the theorem.

According to T. L. Hayden and H. C. Howard [7, Theorem 2], equation (1) is oscillatory if

$$
\inf _{\|\alpha\|=1}\left(\left[\int_{0}^{x} P(t) d t\right] \alpha, \alpha\right) \rightarrow \infty \quad \text { as } \quad x \rightarrow \infty .
$$

This is equivalent to saying that the minimum eigenvalue $\lambda_{0}(x)$ of $\int_{0}^{x} P(t) d t$ has the property $\lambda_{0}(x) \rightarrow \infty$ as $x \rightarrow \infty$. Howard [9, Theorem 1] established the same result in the finite dimensional, or matrix, case, i.e., $\mathscr{H}=R^{n}$, Euclidean $n$-space. If we fix any vector $\alpha \in \mathscr{H},\|\alpha\|=1$, then $\alpha$ determines a positive linear functional $g_{\alpha}$ (see identity (5)), and

$$
\lambda_{0}(x) \leqq\left(\left[\int_{0}^{x} P(t) d t\right] \alpha, \alpha\right)=g_{\alpha}\left[\int_{0}^{x} P(t) d t\right] .
$$

Thus the results of Hayden and Howard are special cases of our theorem.

W. Allegretto and L. Erb [1] developed oscillation criteria for the matrix differential system

$$
\left(R(x) Y^{\prime}\right)^{\prime}+P(x) Y=0
$$

where $R$ and $P$ are symmetric with $R$ positive definite on $R^{+}$, and their result specialized to the matrix version of equation (1) is:

THEOREM A. ([1, Corollary 1). For a sequence of integers $i_{1}, i_{2}, \cdots, i_{k}$, where $1 \leqq i_{1}<i_{2}<\cdots<i_{k} \leqq n$, let $\Sigma A(x)$ denote the sum of all of the entries of the $k \times k$ submatrix of $A(x)$ obtained by deleting all rows and columns except the $i_{j}, j=1,2, \cdots, k$, rows and columns. Then equation (1) is oscillatory if

$$
\lim _{x \rightarrow \infty} \int_{0}^{x} \Sigma P(t) d t=\infty
$$


If we let $e$ be the $n$-component vector with 1 's in the $i_{1}, i_{2}, \cdots, i_{k}$ positions and 0 's elsewhere, then $g_{e}(\cdot)=(\cdot e, e)$, where $(\cdot, \cdot)$ is the "standard" inner product on $R^{n}$, is a positive functional and $g_{e}(A)=$ $\Sigma A$. Thus Theorem $\mathrm{A}$ is included in Theorem 1 .

Allegretto and Erb's work generalized a result of E. S. Noussair and C. A. Swanson [14], which stated in terms of the matrix version of (1) is:

THEOREM B. Equation (1) is oscillatory if

$$
\lim _{x \rightarrow \infty} \int_{0}^{x} p_{i i}(t) d t=\infty
$$

for some diagonal entry $p_{i i}$ of $P$.

Theorem B extends a result of Etgen [5, Theorem 2] which states that (1), in the matrix case, is oscillatory if

$$
\lim _{x \rightarrow \infty} \int_{0}^{x} \operatorname{tr} P(t) d t=\infty
$$

Each of these results is a special case of Theorem 1. For if $e_{i}$ is the $n$-component vector with a 1 in the $i$ th position and 0 's elsewhere, then $g_{e_{1}}$ is a positive functional on the set of $n \times n$ matrices, and $g_{e_{t}}(A)=$ $\left(A e_{i}, e_{t}\right)=a_{i i}$. Similarly, the functional "trace" is a positive functional on the set of $n \times n$ matrices, and, in fact, for any $n \times n$ matrix $A$,

$$
\operatorname{tr} A=\sum_{i=1}^{n} g_{e_{i}}(A)
$$

Finally, it is clear that Theorem 1 is a generalization of the well-known Leighton-Wintner [12], [18] oscillation theorem for the scalar equation (6).

The work of Hayden and Howard [7, Theorem 3] suggests the following extension of Theorem 1 .

THEOREM 2. If there exists a function $\alpha \in \mathscr{C}\left(R^{+}\right)$such that $\alpha>0$ on $R^{+}$and $\lim _{x \rightarrow \infty} \int_{0}^{x} \alpha^{-1}(t) d t=\infty$, and if the operator $J$, defined by

$$
J(x)=\int_{0}^{x}\left\{\alpha(t) P(t)-\left(\left[\alpha^{\prime}(t)\right]^{2} / 4 \alpha(t)\right) I\right\} d t+\frac{\alpha^{\prime}(t)}{2} I
$$

has the property that there exists an element $g \in \mathscr{G}$ such that $\lim _{x \rightarrow \infty} g[J(x)]=\infty$, then equation (1) is oscillatory. 
Proof. The method of proof is analogous to that of Theorem 1, and so we shall omit most of the details.

Assume the theorem is false and let $Y$ be any nontrivial conjoined solution of (1). Then $Y$ is nonsingular on the interval $[b, \infty)$ for some $b \in R^{+}$, and the operator

$$
\frac{1}{\alpha(x)} S(x)=-Y^{\prime}(x) Y^{-1}(x)
$$

exists on $[b, \infty)$. Differentiation of this equation, algebraic manipulation of the resulting equation, followed by integration yields

$$
S(x)+\frac{\alpha^{\prime}(x)}{2} I=S(b)+\int_{b}^{x} \frac{1}{\alpha(t)}\left[S(t)+\frac{\alpha^{\prime}(t)}{2} I\right]^{2} d t+J(x)-J(b) .
$$

Define the operator $W$ on $[b, \infty)$ by

$$
W(x)=\int_{b}^{x} \frac{1}{\alpha(t)}\left[S(t)+\frac{\alpha^{\prime}(t)}{2} I\right]^{2} d t .
$$

Now, by hypothesis there exists $g \in \mathscr{G}$ such that $\lim _{x \rightarrow \infty} g[J(x)]=$ $\infty$. Thus there is a point $c \geqq b$ such that

$$
g\left[S(x)+\frac{\alpha^{\prime}(x)}{2} I\right]>g[W(x)] \quad \text { on } \quad[c, \infty)
$$

By differentiating $W$, and making use of the properties of $g$ as illustrated in the proof of Theorem 1 , we find that $g[W(x)]>0$ on $[c, \infty)$ and obtain the inequality

$$
\frac{\{g[W(x)]\}^{\prime}}{\{g[W(x)]\}^{2}}>\frac{\rho}{\alpha(x)} \quad \text { on } \quad[c, \infty)
$$

Integration of this inequality yields

$$
\frac{1}{g[W(c)]}-\frac{1}{g[W(x)]}>\rho \int_{c}^{x} \frac{1}{\alpha(t)} d t
$$

and contradicts the hypothesis $\int_{0}^{\infty} \alpha^{-1}(x) d x=\infty$.

Theorem 2 generalizes the result of Hayden and Howard [7, Theorem 3], as well as that of Etgen [5, Theorem 3]. In the matrix case, if we let $\alpha(x)=x$ and let $g_{e}$ be the positive functional defined by 
$g_{e}(A)=\Sigma A$ in the notation of Theorem $\mathrm{A}$, then Theorem 2 gives the result (again, restricted to equation (1)) of Allegretto and Erb [1, Corollary 2].

3. Weighted averages. In [3], W. J. Coles introduced the idea of weighted averages to obtain an oscillation criterion for the scalar equation (6) which extended a result of P. Hartman [6]. Coles' work was subsequently generalized by J. W. Macki and J. S. W. Wong [13] through the use of averaging pairs. In this section we use averaging pairs to establish for equation (1) the analogue of Macki and Wong's oscillation criterion [13, Theorem 2].

Definition. A pair of real-valued functions $(\sigma, \alpha)$ is an averaging pair if and only if

(i) $\sigma \in \mathscr{C}\left(R^{+}\right), \sigma \geqq 0, \alpha>0$, and $\alpha$ is absolutely continuous on compact subintervals of $R^{+}$; and

(ii) $\lim _{x \rightarrow \infty} \int_{0}^{x} \sigma(t)\left[\int_{0}^{t} \alpha(s) \sigma^{2}(s) d s\right]^{-1} d s=\infty$.

THEOREM 3. If there exists an averaging pair $(\sigma, \alpha)$, and an element $g \in \mathscr{G}$ such that

$$
\lim _{x \rightarrow \infty} g\left[\sum_{x} J(x)\right]=\infty
$$

where $J$ is given by (10) and $\Sigma_{x}: \mathscr{B} \rightarrow \mathscr{B}$ is the linear operator defined by

$$
\sum_{x} A(x)=\left[\int_{0}^{x} \sigma(t) d t\right]^{-1} \int_{0}^{x} \sigma(t) A(t) d t,
$$

then equation (1) is oscillatory.

Remarks. If we take $\sigma \equiv \alpha \equiv 1$ in Theorem 3 , we get that

$$
\lim _{x} g\left[\frac{1}{x} \int_{0}^{x}\left\{\int_{0}^{t} P(s) d s\right\} d t\right]=\infty
$$

implies (1) is oscillatory. This is a generalization of Hartman's result [6]. Since (7) implies (13), Theorem 3 implies Theorem 1 in this case. By restricting to averaging pairs of the form $(1 / \alpha, \alpha)$, where $\lim _{x \rightarrow \infty} \int_{0}^{x} \alpha^{-1}(t) d t=\infty$, we obtain an extension of Theorem 2. Finally, averaging pairs of the form $(\sigma, 1)$ provide a generalization of Cole's result [3, Theorem 1]. 
Proof of Theorem 3. Let $(\sigma, \alpha)$ be an averaging pair and let $g \in \mathscr{G}$ have the property $\lim _{x \rightarrow \infty} g\left[\Sigma_{x} J(x)\right]=\infty$. Assume that the theorem is false, and let $Y$ and $S$ be the operators specified in the proof of Theorem 2. Consider equation (11) and let $V(x)=S(x)+\left(\alpha^{\prime}(x) / 2\right) I$ to obtain

$$
V(x)=\int_{b}^{x} \alpha^{-1}(t) V^{2}(t) d t+J(x)+S(b)-J(b) .
$$

By applying the operator $\Sigma_{x}$, we get

$$
\sum_{x} V(x)=\sum_{x} \int_{b}^{x} \alpha^{-1}(t) V^{2}(t) d t+\sum_{x} J(x)+S(b)-J(b),
$$

Since $g\left[\sum_{x} J(x)\right] \rightarrow \infty$ as $x \rightarrow \infty$, it follows that

$$
g\left[\sum_{x} V(x)\right]>g\left[\sum_{x} \int_{b}^{x} \alpha^{-1}(t) V^{2}(t) d t\right]>0
$$

on $[c, \infty)$ for some $c \geqq b$, which implies

$$
g\left[\int_{b}^{x} \sigma(t) V(t) d t\right]>g\left[\int_{b}^{x} \sigma(t) \int_{b}^{t} \alpha^{-1}(s) V^{2}(s) d s d t\right]>0
$$

on $[c, \infty)$. By squaring both sides of (14) and using the linearity of $g$, we have

$$
\left\{\int_{b}^{x} \sigma(t) \alpha^{1 / 2}(t) g\left[\alpha^{-1 / 2}(t) V(t)\right] d t\right\}^{2}>\left\{g\left[\int_{b}^{x} \sigma(t) \int_{b}^{t} \alpha^{-1}(s) V^{2}(s) d s d t\right]\right\}^{2}
$$

The Cauchy-Schwartz inequality, together with the properties of $g$, namely (4), applied to the left side of this inequality produces the inequality

$$
\begin{array}{r}
\frac{1}{\rho}\left\{\int_{b}^{x} \sigma^{2}(t) \alpha(t) d t\right\}\left\{\int_{b}^{x} g\left[\alpha^{-1}(t) V^{2}(t)\right] d t\right\} \\
>\left\{g\left[\int_{b}^{x} \sigma(t) \int_{b}^{t} \alpha^{-1}(s) V^{2}(s) d s d t\right]\right\}^{2}
\end{array}
$$

on $[c, \infty)$.

Define the operator $W$ on $[b, \infty)$ by

$$
W(x)=\int_{b}^{x} \sigma(t) \int_{b}^{t} \alpha^{-1}(s) V^{2}(s) d s d t .
$$


Then, from (14), $g[W(x)]>0$ on $[c, \infty)$. The derivative of $W$ is

$$
W^{\prime}(x)=\sigma(x) \int_{b}^{x} \alpha^{-1}(t) V^{2}(t) d t
$$

so that

$$
\{g[W(x)]\}^{\prime}=g\left[W^{\prime}(x)\right]=\sigma(x) \int_{b}^{x} g\left[\alpha^{-1}(t) V^{2}(t)\right] d t
$$

If we multiply (15) by $\sigma(x)$ and write the resulting inequality in terms of the operator $W$, we get

$$
\frac{1}{\rho}\left\{\int_{b}^{x} \sigma^{2}(t) \alpha(t) d t\right\}\{g[W(x)]\}^{\prime}>\sigma(x)\{g[W(x)]\}^{2}
$$

or

$$
\frac{\{g[W(x)]\}^{\prime}}{\{g[W(x)]\}^{2}}>\rho \cdot \sigma(x)\left[\int_{b}^{x} \sigma^{2}(t) \alpha(t) d t\right]^{-1}
$$

Integration of this inequality together with condition (ii) of the Definition produces the desired contradiction.

4. Nonlinear systems. Let $P: R^{+} \times \mathscr{B} \times \mathscr{B} \rightarrow \mathscr{B}$ be continuous, and consider the second order "nonlinear" differential operator $L$ defined by

$$
L[Y]=Y^{\prime \prime}+P\left(x, Y, Y^{\prime}\right) Y
$$

Nonlinear differential systems of the form

$$
L[Y]=0
$$

as well as differential inequalities of the form

$$
L[Y] \leqq 0,
$$

have been considered by a number of authors. See, for example, [4], [5], [10], [11], [14], [16], and [17]. An examination of these results shows that the nonlinear systems are defined in a manner such that the methods developed for linear differential systems can be applied. In this sense, then, the results presented in this paper can be extended to both (16) and (17). 
Let $\Gamma$ denote the collection of functions $Y: R^{+} \rightarrow \mathscr{B}$ such that $Y$ is twice differentiable, $Y^{*} Y^{\prime}=Y^{* \prime} Y$ on $R^{+}$, and $Y(c)$ is nonsingular for at least one $c \in R^{+}$.

We conclude this paper with a statement of the analogue of Theorem 3 for the nonlinear differential inequality (17). The proof of this result is, of course, virtually identical to the proof of Theorem 3.

THEOREM 4. If there exists an averaging pair $(\sigma, \alpha)$, and an element $g \in \mathscr{G}$ such that

$$
\lim _{x \rightarrow \infty} g\left[\sum_{x} K\left(x, Y, Y^{\prime}\right)\right]=\infty
$$

for all $Y \in \Gamma$; where $K: R^{+} \times \mathscr{B} \times \mathscr{B} \rightarrow \mathscr{B}$ is defined by

$$
K\left(x, Y, Y^{\prime}\right)=\int_{0}^{x}\left\{\alpha(t) P\left[t, Y(t), Y^{\prime}(t)\right]-\left(\left[\alpha^{\prime}(t)\right]^{2} / 4 \alpha(t)\right) I\right\} d t+\frac{\alpha^{\prime}(t)}{2} I
$$

and $\Sigma_{x}$ is the operator defined by (12), then there is an oscillatory element $Y \in \Gamma$ such that $Y$ satisfies (17), i.e., (17) is oscillatory.

\section{REFERENCES}

1. W. Allegretto and L. Erb, Oscillation criteria for matrix differential inequalities, Canad. Math. Bull., 16 (1973), 5-10.

2. W. J. Coles, A simple proof of a well-known oscillation theorem, Proc. Amer. Math. Soc., 19 (1968), 507.

3. - An oscillation criterion for second order linear differential equations, Proc. Amer. Math. Soc., 19 (1968), 755-759.

4. G. J. Etgen, Oscillatory properties of certain nonlinear matrix differential systems, Trans. Amer. Math. Soc., 122 (1966), 289-310.

5. - Oscillation criteria for nonlinear second order matrix differential equations, Proc. Amer. Math. Soc., 27 (1971), 259-267.

6. P. Hartman, On nonoscillatory linear differential equations of second order, Amer. J. Math., 74 (1952), 389-400.

7. T. L. Hayden and H. C. Howard, Oscillation of differential equations in Banach spaces, Ann. Mat. Pure Appl., 85 (1970), 383-394.

8. E. Hille, Lectures on Ordinary Differential Equations, Addison-Wesley Publishing Company, Reading, Massachusetts, 1969.

9. H. C. Howard, Oscillation criteria for matrix differential equations, Canad. J. Math., 19 (1967), 184-199.

10. A. G. Kartsatos, Oscillation of nonlinear systems of matrix differential equations, Proc. Amer. Math. Soc., 30 (1971), 97-101.

11. K. Kreith, Oscillation criteria for nonlinear matrix differential equations, Proc. Amer. Math. Soc., 26 (1970), 270-272.

12. W. Leighton, On self adjoint differential equations of second order, J. London Math. Soc., 27 (1952), 37-47. 
13. J. W. Macki and J. S. W. Wong, Oscillation theorems for linear second order ordinary differential equations, Proc. Amer. Math. Soc., 20 (1969), 67-72.

14. E. S. Noussair and C. A. Swanson, Oscillation criteria for differential systems, J. Math. Anal. and Appl., 36 (1971), 575-580.

15. C. E. Rickart, Banach Algebras, D. Van Nostrand Company, Inc., Princeton, New Jersey, 1960.

16. C. A. Swanson, Oscillation criteria for nonlinear matrix differential inequalities, Proc. Amer. Math. Soc., 24 (1970), 824-827.

17. E. C. Tomastik, Oscillation of nonlinear matrix differential equations of second order, Proc. Amer. Math. Soc., 19 (1968), 1427-1431.

18. A. Wintner, A criterion of oscillatory stability, Quart. Appl. Math., 7 (1949), 115-117.

Received March 9, 1976.

UNIVERSITY OF HOUSTON 



\section{Pacific Journal of Mathematics}

\section{Vol. 66, No. $1 \quad$ November, 1976}

Helen Elizabeth. Adams, Factorization-prime ideals in integral domains ............ Patrick Robert Ahern and Robert Bruce Schneider, The boundary behavior of Henkin's kernel.

Daniel D. Anderson, Jacob R. Matijevic and Warren Douglas Nichols, The Krull

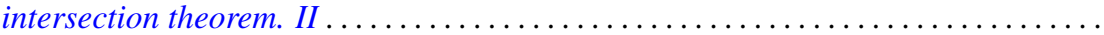

Efraim Pacillas Armendariz, On semiprime P.I.-algebras over commutative regular

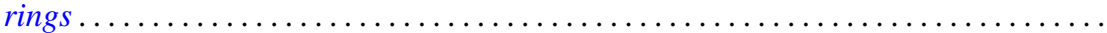

Robert H. Bird and Charles John Parry, Integral bases for bicyclic biquadratic fields

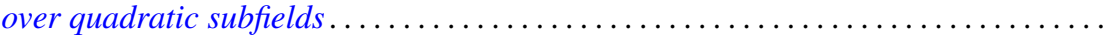

Tae Ho Choe and Young Hee Hong, Extensions of completely regular ordered

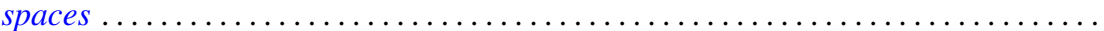

John Dauns, Generalized monoform and quasi injective modules ...............

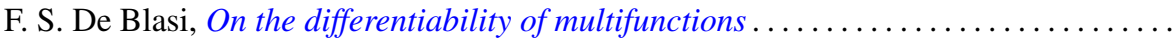

Paul M. Eakin, Jr. and Avinash Madhav Sathaye, R-endomorphisms of $R[[X]]$ are

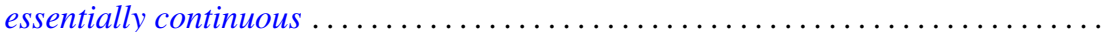

Larry Quin Eifler, Open mapping theorems for probability measures on metric

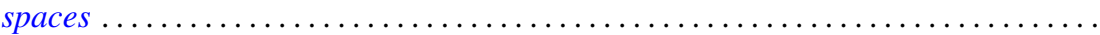

Garret J. Etgen and James Pawlowski, Oscillation criteria for second order self adjoint

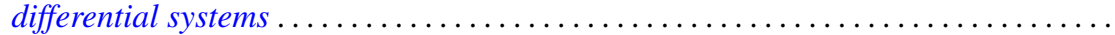

Ronald Fintushel, Local $S^{1}$ actions on 3-manifolds .

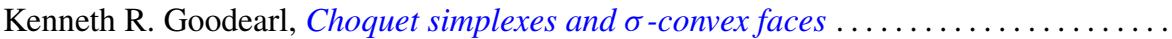

John R. Graef, Some nonoscillation criteria for higher order nonlinear differential

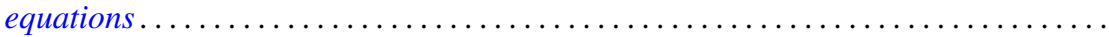

Charles Henry Heiberg, Norms of powers of absolutely convergent Fourier series: an example.

Les Andrew Karlovitz, Existence of fixed points of nonexpansive mappings in a space

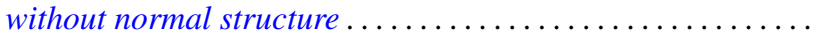

Gangaram S. Ladde, Systems of functional differential inequalities and functional

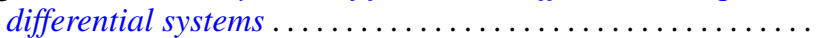

Joseph Michael Lambert, Conditions for simultaneous approximation and interpolation with norm preservation in $C[a, b]$.

Ernest Paul Lane, Insertion of a continuous function.

Robert F. Lax, Weierstrass points of products of Riemann surfaces .

Dan McCord, An estimate of the Nielsen number and an example concerning the Lefschetz fixed point theorem...

Paul Milnes and John Sydney Pym, Counterexample in the theory of continuous functions on topological groups...

Peter Johanna I. M. De Paepe, Homomorphism spaces of algebras of holomorphic functions

Judith Ann Palagallo, A representation of additive functionals on $L^{p}$-spaces,

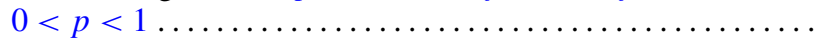

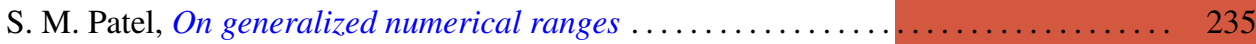

Thomas Thornton Read, A limit-point criterion for expressions with oscillatory

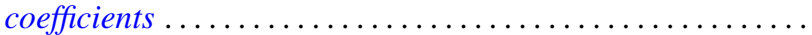

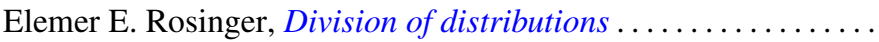

Peter S. Shoenfeld, Highly proximal and generalized almost finite

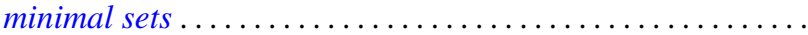

R. Sirois-Dumais and Stephen Willard, Quotient-universal sequential spaces

Robert Charles Thompson, Convex and concave functions of singular values of matrix sums....

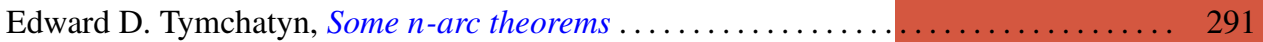

